\title{
Marantic Endocarditis Associated with COVID-19: A Rare Case Report of a Potentially Deadly Disease
}

\author{
$\underline{\text { Kok Hoe Chan }}^{1}$, Ormena Joseph ${ }^{1}$, Eyad Ahmed ${ }^{1}$, Apoorva Kommidi ${ }^{1}$, Addi Suleiman ${ }^{1,2}$, Maria E Szabela ${ }^{1,3}$, Jihad Slim ${ }^{1,3}$ \\ ${ }^{1}$ Department of Medical Education, Saint Michael's Medical Centre, New York Medical College, Newark, New Jersey, USA \\ ${ }^{2}$ Department of Cardiology, Saint Michael's Medical Centre, New York Medical College, Newark, New Jersey, USA \\ ${ }^{3}$ Department of Infectious Disease, Saint Michael's Medical Centre, New York Medical College, Newark, New Jersey, USA
}

\section{Doi: 10.12890/2021_002409 - European Journal of Case Reports in Internal Medicine - ๑ EFIM 2020}

Received: 20/02/2021

Accepted: 02/03/2021

Published: 19/03/2021

\begin{abstract}
How to cite this article: Chan KH, Joseph O, Ahmed E, Kommidi A, Suleiman A, Szabela ME, Slim J. Marantic endocarditis associated with COVID-19: a rare case report of a potentially deadly disease. EJCRIM 2021;8: doi:10.12890/2021_002409
\end{abstract}

Conflicts of Interests: The Authors declare that there are no competing interests.

This article is licensed under a Commons Attribution Non-Commercial 4.0 License

\section{ABSTRACT}

Coronavirus disease 2019 (COVID-19) has overwhelmed healthcare globally with millions of cases and over 2 million deaths worldwide. The hypercoagulable state associated with COVID-19 is a well-recognized complication that carries a poor prognosis. Marantic endocarditis, or non-bacterial thrombotic endocarditis (NBTE), is caused by a sterile vegetation on the cardiac valves resulting from the deposition of fibrin and platelet aggregates. It is highly associated with the hypercoagulable and acquired inflammatory states. Herein, we report a unique and rare case of COVID-19 presenting with NBTE.

\section{LEARNING POINTS}

- COVID-19 has caused a major global pandemic, with high morbidity and mortality.

- One of the complications of COVID-19 infection is a hypercoagulable state.

- To the best of our knowledge, we present only the second case of marantic endocarditis in a patient with COVID-19, in the hope of raising awareness among physicians of this potential rare association.

\section{KEYWORDS}

Marantic endocarditis, non-bacterial thrombotic endocarditis, COVID-19, hypercoagulable state, COVID-19 associated coagulopathy

\section{INTRODUCTION}

Marantic endocarditis, also known as non-bacterial thrombotic endocarditis (NBTE), is a rare entity where non-infectious vegetations are attached to the valvular area or endocardium. The condition was first described by Zeigler in $1888^{[1]}$, who reported the deposition of fibrin on heart valves, and subsequently termed 'nonbacterial thrombotic endocarditis' by Gross and Friedberg in $1936^{[2]}$. NBTE is also known as noninfectious endocarditis, and is characterized by the presence of vegetations on the cardiac valves. These vegetations are mainly composed of platelet aggregates and fibrin, and are not infectious; they do not contain bacteria ${ }^{[3]}$. NBTE is mainly associated with hypercoagulable states or malignancy and typically affects the aortic or mitral valves ${ }^{[4]}$.

Coronavirus disease 2019 (COVID-19) has caused a major pandemic, with high morbidity and mortality. One of the complications of this infection is a hypercoagulable state, with deep vein thrombosis and pulmonary embolism the most common thromboembolic events reported in the literature ${ }^{[5]}$. We describe a unique and rare case of COVID-19 presenting with NBTE. 


\section{CASE DESCRIPTION}

A 61-year-old man with a significant medical history of seizure/epilepsy and non-ischaemic heart failure with a reduced ejection fraction (HFrEF), who was also an active smoker, presented to our hospital with weakness and non-productive cough of 1 weeks' duration. He was homeless and poorly compliant with his medications. His initial vital signs showed a temperature of $37.1^{\circ} \mathrm{C}$, heart rate of $92 / \mathrm{min}$, tachypnoea with a respiratory rate of $23 / \mathrm{min}$, oxygen saturation of $87 \%$ on room air and blood pressure of $103 / 65 \mathrm{mmHg}$. Physical examination revealed decreased air entry to the lungs bilaterally with diffuse crackles and rhonchi. Cardiovascular examination revealed a systolic murmur best heard at the aortic area with no radiation to the carotid, and with an otherwise regular rate and rhythm. He had +1 bilateral lower limb

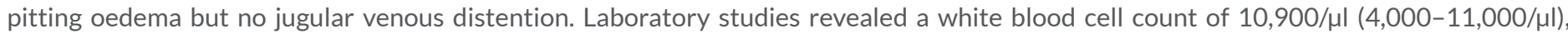
microcytic anaemia with haemoglobin $10.7 \mathrm{~g} / \mathrm{dl}$ (13.5-16 g/dl) and a mean corpuscular volume of $74 \mathrm{fl}$ (81.2-95.1 fl), and thrombocytopenia

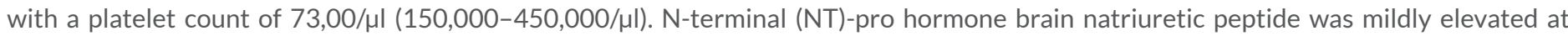
517 pg/ml (0-100 pg/ml). Inflammatory markers were markedly elevated with D-dimer 50,047 ng/ml (0-500 ng/ml), ferritin 744.7 ng/ml (24-336 ng/ml), C-reactive protein $12.2 \mathrm{mg} / \mathrm{dl}(0.0-0.8 \mathrm{mg} / \mathrm{dl})$ and lactate dehydrogenase $592 \mathrm{U} / \mathrm{l}$ (122-222 U/l). The iron profile showed iron $17 \mu \mathrm{g} / \mathrm{dl}(50-150 \mu \mathrm{g} / \mathrm{dl})$, iron saturation 12.4\% (20-55\%) and TIBC $137 \mu \mathrm{g} / \mathrm{dl}$ (250-400 $\mu \mathrm{g} / \mathrm{dl})$. The haemolysis profile was notable for high LDH, and normal haptoglobin and reticulocyte counts. Schistocytes and platelet clumping were seen in a peripheral blood smear. SARS-CoV-2 RT-PCR from a nasopharyngeal swab was positive. The electrocardiograph (ECG) showed sinus tachycardia with signs of left ventricular hypertrophy. Chest x-ray (CXR) showed multifocal infiltrates consistent with COVID-19 infection (Fig. 1). A CT angiogram of the chest revealed right upper lobe segmental and subsegmental emboli, suggestions of multiple thrombi in the left ventricle, and multi-lobar predominantly peripheral ground-glass opacities and consolidation (Fig. 2). An echocardiogram revealed an ejection fraction of 20-25\% with signs of left ventricular hypertrophy, no right heart strain, and aortic valve vegetation (Fig. 3). A CT scan of the head did not show any emboli. Blood cultures were persistently negative. In light of the high risk of vegetations/thrombi showering to the brain, the patient was started on a therapeutic dose of enoxaparin. At the same time, he was also receiving guideline-directed medical therapy for heart failure and supportive treatment for COVID-19.
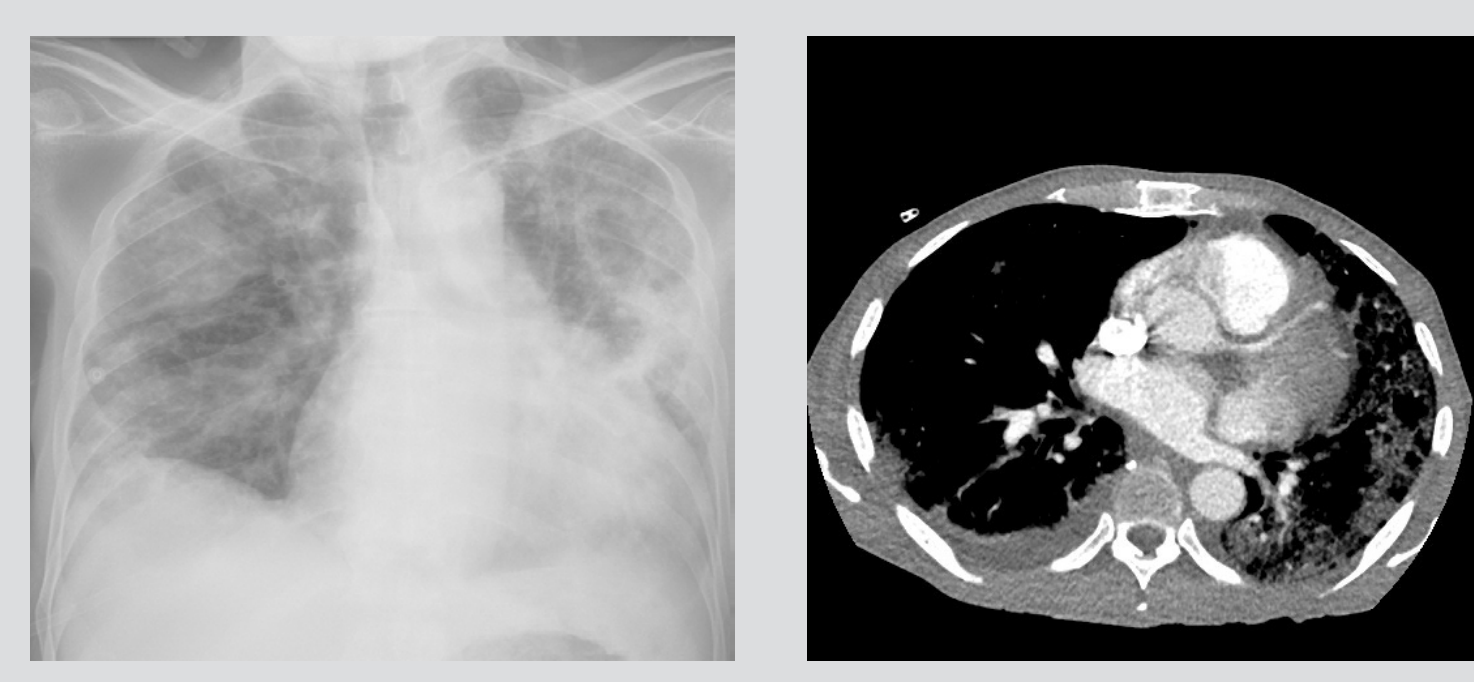

Figure 1. Chest $x$-ray showing multifocal infiltrates consistent with COVID-19 infection

Figure 2. CT angiogram of the chest revealing right upper lobe segmental and subsegmental emboli, and multi-lobar predominantly peripheral ground glass opacities and consolidation
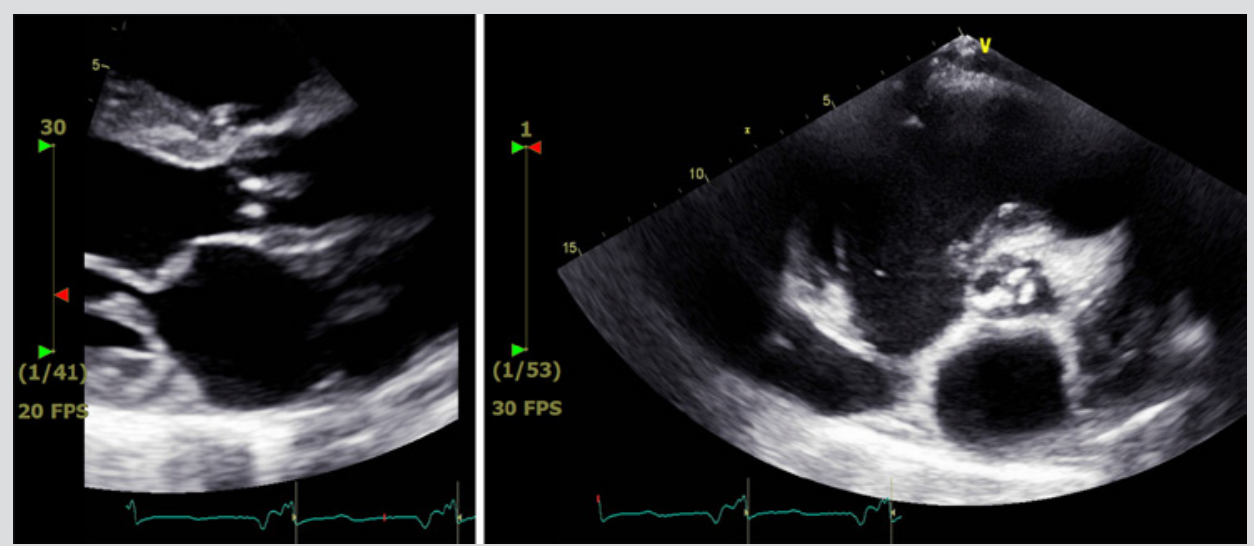

Figure 3. Echocardiogram showing aortic valve vegetation 
Upon discharge, the patient was transitioned to a therapeutic dose of apixaban. A full hypercoagulability investigation showed mildly elevated anticardiolipin antibodies. Other hypercoagulable tests and autoimmune work-up were unremarkable. The patient was seen in the outpatient clinic 4 weeks after discharge from hospital, where his echocardiogram showed resolution of the vegetation and left ventricle thrombus.

\section{DISCUSSION}

NBTE is a relatively rare condition most commonly found in patients with underlying malignancy or systemic lupus erythematosus ${ }^{[4]}$. Other conditions associated with NBTE include systemic or acquired inflammatory conditions such as rheumatoid arthritis and sepsis, connective tissue disorders, autoimmune disorders and other hypercoagulable states but not COVID-19[6]. A diagnosis of NBTE is most frequently made at autopsy, with incidence ranging from $0.3 \%$ to $9.3 \%$ in the adult populatio ${ }^{[1]}$. Although all age groups can be affected, NBTE is usually reported in patients between the fourth and eighth decades of life ${ }^{[1,7,8]}$. There is no gender predilection ${ }^{[7,8]}$.

Although the pathogenesis of NBTE remains unclear, it is similar to the process by which underlying malignancy or a high inflammatory state results in elevated levels of cytokines (tumour necrosis factor and interleukin-1), which in turn causes local tissue damage with activation of the coagulation cascade resulting in vegetation formation ${ }^{[9]}$. In our patient, COVID-19 infection causing a high inflammatory state with cytokine storm possibly contributed to the local tissue damage and in turn caused thrombus vegetation formation on the disrupted areas. These sterile vegetations have reduced cellular organization at the deposition site, which makes them friable and readily prone to detachment with subsequent systemic embolization ${ }^{[10]}$. Balata et al. reported a case of NBTE with COVID-19 in a 76-year-old woman with systemic cerebral embolism, who was found to have two small vegetations on her mitral valve, visualized only with a transoesophageal echocardiogram ${ }^{[11]}$. In contrast, our patient had the echocardiogram as part of the investigations of the murmur heard on physical examination, with visualization of the vegetation on trans-thoracic echocardiogram. To the best of our knowledge, we are one of the few authors who have reported one of these rare cases.

Patients with NBTE should be treated with lifelong anticoagulation due to the high risk of systemic embolism and recurrent thromboembolism ${ }^{[12]}$. Recommendations for the treatment of malignancy-associated NBTE include therapeutic anticoagulation with heparin, and treatment of the underlying malignancy. Warfarin has been found to be ineffective in preventing recurrent thromboembolic events in NBTE ${ }^{[13]}$. The optimum treatment of the hypercoagulable state in patients with COVID-19 remains unclear. A small randomized phase II clinical trial (HESACOVID) randomly assigned 20 individuals with severe COVID-19 to receive therapeutic-dose anticoagulation (enoxaparin $1 \mathrm{mg} / \mathrm{kg}$ twice daily) or prophylactic-dose anticoagulation (enoxaparin, $40 \mathrm{mg}$ once daily or unfractionated heparin, 5000 units three times daily), with appropriate adjustments for age, weight and kidney function. It was shown that therapeutic enoxaparin decreased the need for mechanical ventilation in severe COVID-19[14]. Our patient was treated with therapeutic enoxaparin and upon discharge was transitioned to a therapeutic dose of apixaban with resolution of his vegetation after a month of therapy. The plan is to continue anticoagulation for life.

\section{CONCLUSION}

In conclusion, we presented a case of NBTE in a patient with COVID-19, which we propose may have been caused by the underlying hypercoagulable or inflammatory state. NBTE carries a high risk of systemic embolism and recurrent thromboembolism, so prompt treatment with anticoagulation is paramount. We have described this case to highlight the potential association of COVID-19 with this serious condition in order to raise awareness among physicians. 


\section{REFERENCES}

1. Lopez JA, Ross RS, Fishbein MC, Siegel RJ. Nonbacterial thrombotic endocarditis: a review. Am Heart J 1987;113(3):773-784. doi: 10.1016/0002-8703(87)90719-8. PMID: 3548296.

2. Gross L, Friedberg CK. Nonbacterial thrombotic endocarditis: classification and general description. Arch Intern Med (Chic) 1936;58(4):620-640. doi: 10.1001/ archinte.1936.00170140045004

3. AsopaS, Patel A, Khan OA, Sharma R, OhriSK. Non-bacterial thrombotic endocarditis. Eur J Cardiothorac Surg 2007;32(5):696-701. https://doi.org/10.1016/j.ejcts.2007.07.029

4. Deppisch LM, Fayemi AO. Non-bacterial thrombotic endocarditis: clinicopathologic correlations. Am Heart J 1976;92(6):723-729. doi: 10.1016/s0002-8703(76)80008-7. PMID: 998478

5. Bilaloglu S, Aphinyanaphongs Y, Jones S, Iturrate E, Hochman J, Berger JS. Thrombosis in hospitalized patients with COVID-19 in a New York City health system. JAMA 2020;324:799.

6. Hojnik M, George J, Ziporen L, Shoenfeld Y. Heart valve involvement (Libman-Sacks endocarditis) in the antiphospholipid syndrome. Circulation 1996;93(8):1579-1587. doi: 10.1161/01.cir.93.8.1579. PMID: 8608627.

7. Kuramoto K, Matsushita S, Yamanouchi H. Nonbacterial thrombotic endocarditis as a cause of cerebral and myocardial infarction. Jpn Circ J 1984;48(9):1000-1006. doi: 10.1253/jcj.48.1000. PMID: 6481938.

8. Macdonald RA, Robbins SL. The significance of nonbacterial thrombotic endocarditis: an autopsy and clinical study of 78 cases. Ann Intern Med 1957;46(2):255-273. doi: 10.7326/0003-4819-46-2-255. PMID: 13403513.

9. Bick RL. Cancer-associated thrombosis. N Engl J Med 2003;349:109-111. doi: 10.1056/NEJMp030086. PMID: 12853582.

10. Shatila W, Rizkallah A, Saad Aldin E, Tfayli A. Nonbacterial thrombotic endocarditis as the sole manifestation of stage IV gastric cancer. J Med Case Reports 2014;8:267. doi: 10.1186/1752-1947-8-267. PMID: 25091999. PMCID: PMC4131800.

11. Balata D, Mellergård J, Ekqvist D, Baranowski J, Garcia IA, Volosyraki M, et al. Non-bacterial thrombotic endocarditis: a presentation of COVID-19. Eur J Case Rep Intern Med 2020;7(8):001811. doi: 10.12890/2020_001811

12. Rogers LR, Cho ES, Kempin S, Posner JB. Cerebral infarction from non-bacterial thrombotic endocarditis. Clinical and pathological study including the effects of anticoagulation. Am J Med 1987;83(4):746-756. doi: 10.1016/0002-9343(87)90908-9. PMID: 3674060.

13. el-Shami K, Griffiths E, Streiff M. Nonbacterial thrombotic endocarditis in cancer patients: pathogenesis, diagnosis, and treatment. Oncologist 2007;12(5):518-523. doi: 10.1634/theoncologist.12-5-518. PMID: 17522239.

14. Lemos ACB, do Espírito Santo DA, Salvetti MC, Gilio RN, Agra LB, Pazin-Filho A, Miranda CH. Therapeutic versus prophylactic anticoagulation for severe COVID-19: a randomized phase II clinical trial (HESACOVID). Thromb Res 2020;196:359-366. doi: 10.1016/j.thromres.2020.09.026. PMID: 32977137. PMCID: PMC7503069. 